Session 2620

\title{
Controlling a Mobile Robot with a Personal Digital Assistant
}

\author{
Robert L. Avanzato \\ Penn State Abington
}

\begin{abstract}
Penn State Abington has been exploring the application of personal digital assistant (PDA) technology to the control of autonomous mobile robots. The PDA is an inexpensive, handheld computer that supports a wide range of mobile software applications, and features serial and infrared communication capabilities. Undergraduate students have participated in an applied research effort to develop PDA-controlled autonomous robots for several robot design competitions. One of the innovative aspects of the handheld-based robot is that the technology allows students to enter and modify software directly on the handheld computer. This feature is particularly useful in environments where conventional desktop and laptop computers are not conveniently available. This paper will describe the architecture of the robots and discuss the educational benefits of the handheld approach to mobile robot control.
\end{abstract}

\subsection{Introduction}

Starting in the fall of 1999, Penn State Abington has been actively exploring the integration of Palm ${ }^{\mathrm{TM}}$ [1] handheld computers (personal digital assistants) into select undergraduate courses in the areas of Information Sciences and Technology, computer engineering, French language instruction, and mobile robot design. The personal digital assistant (PDA) is a handheld, mobile computer that supports database, spreadsheet, document viewing, graphics, programming, and web-browsing software. Additionally, the PDA features both serial and infrared communication capabilities. The primary focus of these integration efforts is to promote active and collaborative learning in the classroom and laboratory settings, and to explore enhancements and efficiencies in the delivery of instruction [2].

A team of sophomore computer science majors at Penn State Abington participated in a research effort in the spring of 2000 to explore the application of personal digital assistant technology to mobile robot design and operation. There were three major thrusts to the experimental work. In the first area of investigation, the Palm unit was utilized as a programmable IR remote controller used to direct and program the motion of existing mobile robots. The emphasis here was to design a meaningful graphical user interface on the Palm PDA to effectively control a mobile robot.

The second area of investigation explored the application of the Palm PDA as a terminal emulator to communicate with a mobile robot via a serial connection. Commands could be issued to the robot, and data that was collected by the robot can be 
uploaded to the Palm device for analysis. The motivation behind this activity is to provide an inexpensive, handheld interface to a mobile robot in the field.

In the third area of investigation, which will be the focus of this paper, the Palm device served as the "brain" of an autonomous mobile robot. In this configuration, the Palm PDA was resident on the robot platform, and was interfaced to a commercially available controller board through a serial connection. This arrangement allowed the PDA to control motors and actuators and also to receive data from a variety of sensors located on the robot. The prototype robot was designed to perform in a regional firefighting robot contests in the spring of 2000 [3,4]. During the fall of 2000, a second Palm-controlled autonomous robot was designed for participation in a regional robot navigation contest hosted by Penn State Abington [3].

One of the innovative aspects of the PDA-based robot was that the technology allowed students to enter and modify control software directly on the handheld computer. This was found to be particularly useful in environments, such as at a contest site or in the field, where conventional desktop and laptop computers are not conveniently available. Another important result of this research is that the handheld programming environment facilitated collaborative programming. That is, a student could write or modify a segment of robot control software directly on a PDA, and then electronically "beam" this software to another handheld, where it could be merged with other software components and eventually be executed by the robot.

The research efforts outlined above were incorporated into robotics workshop activities at the Pennsylvania Governor's School of Information Technology held at Penn State University Park in the summer of 2000. A demonstration of mobile robots interfaced to Palm PDA technology was also presented at a National Governor's Association Conference event during the same time period.

The remainder of this paper will describe the architecture of the Penn State PDAbased robot prototypes, the programming development strategy, and discuss the educational benefits of the handheld approach to mobile robot control.

\subsection{Palm Robot Architecture}

The Palm-based autonomous robot designed at Penn State Abington for a fire-fighting robot contest uses a modified $\mathrm{R} / \mathrm{C}$ treaded platform as shown in figure 1. A Palm IIIx PDA is connected directly to a Pontech SV203 controller board [5] through a null modem serial connection. Under software control, the Palm sends ASCII character data to the controller board to control motors and to receive data from

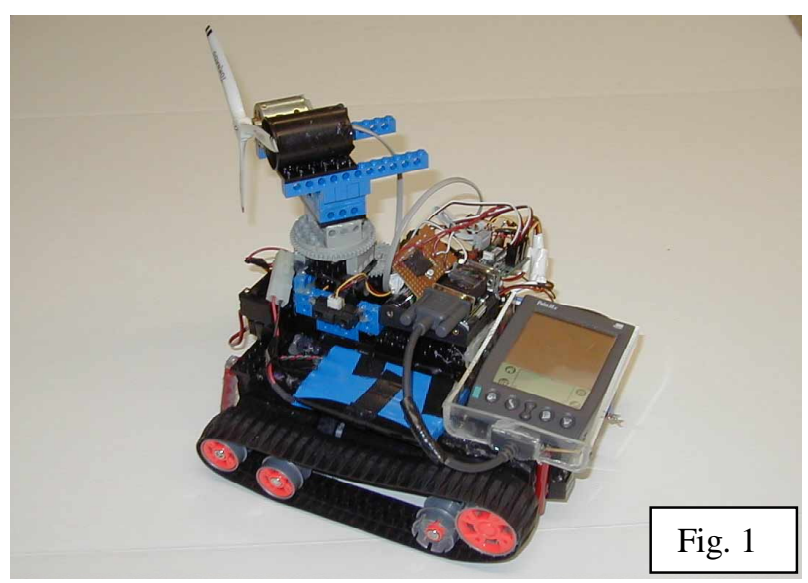
sensors. The Pontech board features a 5-channel, 8-bit analog-to-digital converter (A/D) and can control up to 8 servo motors. The servo motor outputs can optionally be configured to behave as digital outputs. The servo motor outputs are connected to commercially available electronic speed controllers that provide PWM outputs (variable speed) to the DC drive motors. There are two independently powered drive systems on 
this chassis. One drive system allows for motion in the forward and reverse directions, and the other drive system allows for motion side-to-side. Three Sharp (GP2D12) infrared rangefinders were connected to A/D inputs of the Pontech controller board.

The objective of the fire-fighting robot contest is to move autonomously through a walled maze consisting of four rooms, locate a burning candle which has been placed randomly within one of the four rooms, and extinguish the candle in a minimum amount of time. The IR rangefinders provided distance to the walls and were utilized to enable successful navigation through the maze. An inexpensive phototransistor, mounted on a servo motor, was employed to detect the candle flame. Phototransistors were also mounted on the robot frame to detect the white line that was positioned 12 inches from the candle. It was required that the robot be within 12 inches of the candle before extinguishing the flame. A propeller connected to the shaft of a DC motor (also mounted on the servo motor) was responsible for extinguishing the flame. The robot was capable of extinguishing a candle in the Penn State regional fire-fighting contest in March of 2000. (The robot was not successful in qualifying in the Trinity College national firefighting robot contest in April of 2000 due to a sensor failure.)

The second Palm-based robot prototype was designed and developed in the fall of 2000 by a team of undergraduate students in an introductory digital design course.

(Through funding from Palm, Inc., each student in the class was provided with a Palm PDA to investigate and develop educational applications for handheld computers.)
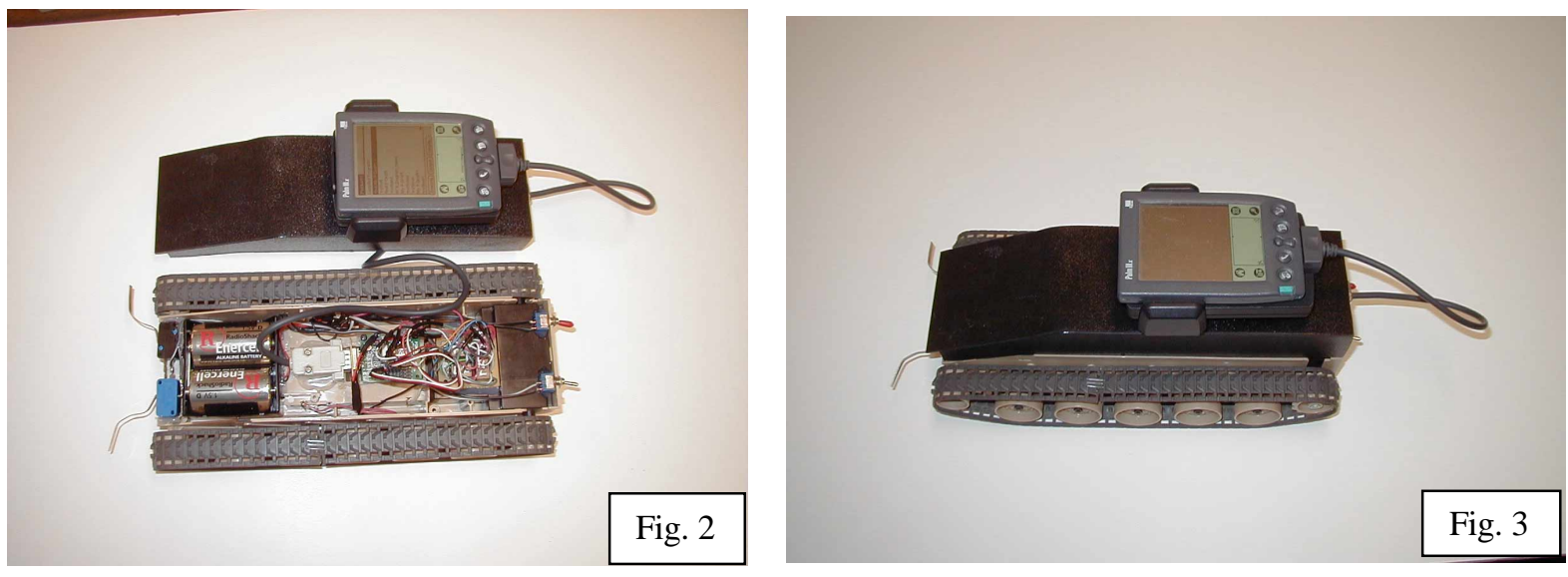

The mobile robot is shown in figures 2 and 3. The Palm PDA is connected to a Pontech controller board as was done with the fire-fighting robot described above. In the case of both robots, the Palm unit can be easily disconnected and removed from the robot. The robot base is a converted toy tank, which measures approximately 12 inches in length. A relay circuit, under software control, provides independent forward and reverse operation for each tread at a constant speed. The robot shown above was equipped with two frontmounted microswitches, which were interfaced to the controller board. The robot was capable of basic obstacle avoidance behavior, and was entered as an exhibition robot at the December 2000 Robo-TrailBlazers autonomous robot contest hosted by Penn State Abington [3].

Both robots described above demonstrate the feasibility of constructing inexpensive Palm PDA-based autonomous mobile robots, within the confines of a course activity, that perform relatively advanced tasks. 


\subsection{Software Development}

One of the key objectives of the PDA-based mobile robot design was to be able to develop and modify software without the use (or with limited use) of a laptop or PC. This is especially useful at a contest site or in the field where these traditional computers are not readily available, or inconveniently located. Autonomous mobile robotics applications often require software adjustments and calibrations to be made at the site of operation. This philosophy can also be extended to the limitations of conventional classrooms and other learning areas that are not equipped with PCs. Utilizing handheld computer technology to support mobile programming would allow mobile robot education to take place in a wider range number of venues.

For these reasons, the software system chosen for this research was PocketC[6]. With this compiler, $\mathrm{C}$ source code can be directly entered and modified in the memo application of the Palm PDA.
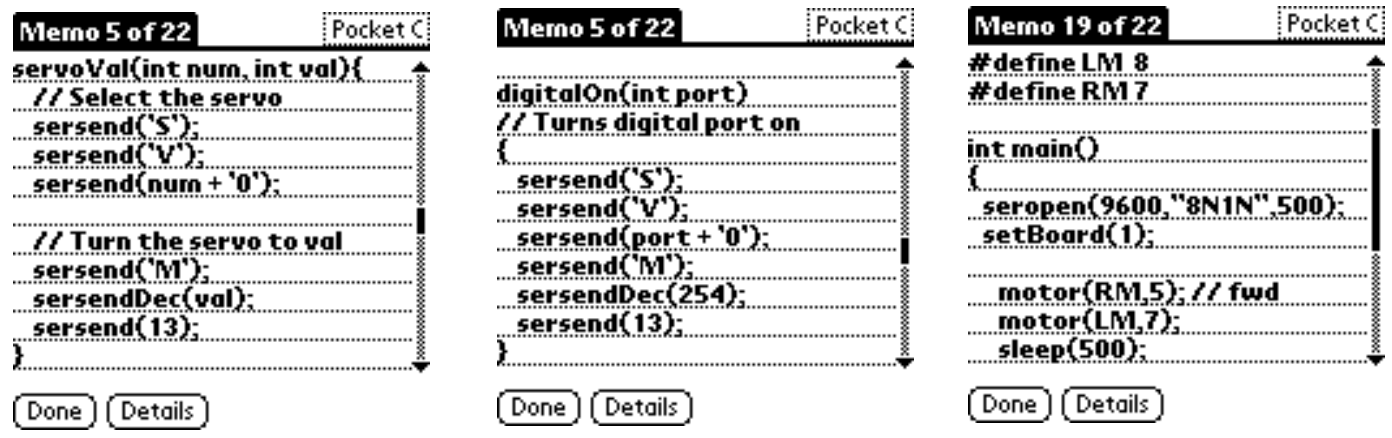

Figure 4. C Source Code

In the case where the source code is large, the file can be initially created on a PC and downloaded onto a Palm device. In other environments, a portable keyboard that is compatible with the Palm PDA can be used to enter software. Figure 4 shows three screenshots of Palm memos containing Pocket $\mathrm{C}$ code. The PocketC product provides support for graphics, sound, databases, and serial I/O. The package is low cost and is intuitive to use. For the robotics projects described above, students were able to write software to interface with the Pontech controller board with little difficulty.

Finally, it should be noted that this software development approach facilitates and encourages a collaborative and team approach to programming. Memo text, containing $\mathrm{C}$ source code, can be beamed through the IR feature from one Palm unit to another. In this way, software programs, functions, and segments can be shared and merged into working programs. The ability to modify text directly on the Palm device, and the capability of electronically sharing software is a key advantage of the PDA-based design. These capabilities are also ideal for integrating robotics programming into a classroom setting.

A Palm-based mobile robot project, PalmPilot Robot Kit (PPRK), has recently been announced by the Robotics Institute of Carnegie Mellon University [7]. This robot features holonomic drive and utilizes the same servo controller board as is used in the Penn State Abington robots. One distinction is the general approach to software. The 
CMU robot kit software focuses on PC-based development tools to create Palm PDA applications, but the hardware can be adapted to alternate software approaches. The commercial availability of this Palm-based robot kit will enhance the accessibility of mobile robotics to a large community.

\subsection{Summary and Conclusions}

This paper has provided an overview of some accomplishments in the application of Palm PDA technology to mobile robotics at Penn State Abington. The two robot projects described in the paper demonstrate the feasibility of mobile robot hardware and software design using the Palm PDA. A key feature of the mobile robot designs described here has been the ability to enter, modify, compile, and execute software directly on the Palm device. This has facilitated a rapid prototyping approach in environments where conventional PCs are not readily available, and has supported collaborative design of software. The integration of PDA technology has a positive impact on mobile robotics in both education and research environments.

\section{Acknowledgements}

The author would like to acknowledge Penn State Abington students Mike Pilquist, Adam Taylor, and Jeffrey Gingerich for their technical contributions to the Palm-based robots. The Palm PDA robotics projects with the CSE 271 digital design class in the Fall of 2000 were supported in part by Palm, Inc.

\section{References}

[1] Palm, Inc., URL:http://www.palm.com

[2] Avanzato, R.L., "Handheld Computers in the Classroom and Laboratory," to appear in the 2001 Annual ASEE Conference Proceedings, Albuquerque, NM, June 2001.

[3] URL: http://www.ecsel.psu.edu/ avanzato/robots

[4] URL: http://www.trincol.edu/ robot

[5] URL: http://www.pontech.com

[6] URL: http://www.orbworks.com

[7] URL: http://www.cs.cmu.edu/ pprk/index.html

\section{BOB AVANZATO}

Bob Avanzato is an associate professor of engineering at the Penn State Abington College. His interests include mobile robotics, fuzzy logic, expert systems, and curricular enhancement. Prior to his position at Penn State, Bob was a senior engineer at the Advanced Technology Laboratories at Martin Marietta where he was involved in digital signal processing and artificial intelligence research and development. (email: rla5@psu.edu) 